\title{
TOEPLITZ OPERATORS AND DIFFERENTIAL EQUATIONS ON A HALF-LINE ${ }^{1}$
}

\author{
J. W. MOELLER
}

ABSTRACT. Let $\mathscr{K}$ be a separable Hilbert space, let $A_{0}, A_{1}, \cdots$, $A_{n}$ denote bounded linear operators from $\mathscr{K}$ into $\mathscr{K}$, and let $\mathscr{D}$ represent the set of all functions in $L^{2}(0, \infty ; \mathscr{K})$ whose first $n$ derivatives belong to $L^{2}(0, \infty ; \mathscr{K})$. Suppose further that the space $\mathscr{H}$ is equipped with an inner product inherited from $L^{2}(0, \infty ; \mathscr{K})$. The main result of this note states that the differential operator

$$
L=A_{n} \frac{d^{n}}{d t^{n}}+A_{n-1} \frac{d^{n-1}}{d t^{n-1}}+\cdots+A_{1} \frac{d}{d t}+A_{0}
$$

acting on $\mathscr{D}$ is continuously invertible if and only if the operator

$$
P(\sigma)=\sum A_{k}^{*} \sigma^{i} \quad(0 \leqq k \leqq n)
$$

acting on the Hilbert space $\mathscr{K}$ has a uniformly bounded inverse everywhere in the open half-plane $\operatorname{Re} \sigma<0$.

Let $\mathscr{K}$ be a separable Hilbert space, and let $A_{0}, A_{1}, \cdots, A_{n}$ denote bounded linear operators from $\mathscr{K}$ into $\mathscr{K}$. In what follows we will obtain necessary and sufficient conditions to insure the continuous invertibility of the differential operator

$$
L=A_{n} \frac{d^{n}}{d t^{n}}+A_{n-1} \frac{d^{n-1}}{d t^{n-1}}+\cdots+A_{1} \frac{d}{d t}+A_{0}
$$

acting on a dense manifold of the Hilbert space $L^{2}(0, \infty ; \mathscr{K})$.

Our approach to this problem is based on the observation that $L$ is unitarily equivalent to a generalized Toeplitz operator. The inversion theory of these operators was recently developed by Rabindranathan [5], who systematically extended the previous work of Widom [7], Devinatz [1], and Pousson [4]. Hereafter we will freely use the terminology, as well as some of the theory, contained in Rabindranathan's paper.

To expose the connections between $L$ and a generalized Toeplitz operator, we first construct a special isometric mapping from $L^{2}(0, \infty ; \mathscr{K})$

Received by the editors December 1, 1971 and, in revised form, March 24, 1972.

AMS (MOS) subject classifications (1970). Primary 34G05; Secondary 47B35.

Key words and phrases. Separable Hilbert space, differential operator, Laguerre function, Toeplitz operator.

1 Work supported by NSF Grant GP-24917.

(c) American Mathematical Society 1973 
onto $H^{2}(\mathscr{K})$, the Hardy space of $\mathscr{K}$-valued analytic functions defined on the interior of the unit disc. This is accomplished by taking the Laguerre functions

$$
g_{n}(t)=\frac{1}{n !} \exp \left(\frac{t}{2}\right) \frac{d^{n}}{d x^{n}}\left[t^{n} \exp (-t)\right]
$$

and then defining $J\left(g_{n} \varphi\right)=z^{n} \varphi, n=0,1, \cdots$, for all $\varphi \in \mathscr{K}$. Since the Laguerre functions constitute an orthonormal basis for $L^{2}(0, \infty)$, the map $J$ may be extended linearly as an isometry from $L^{2}(0, \infty ; \mathscr{K})$ onto $H^{2}(\mathscr{K})$. In the scalar case where $\operatorname{dim} \mathscr{K}=1$, this mapping was employed by the author to study differentiability properties of exponential sums [3], and it also occurs in Rosenblum's earlier work on selfadjoint Toeplitz operators [6].

From the definition of a Laguerre function we deduce that

$$
-2 \frac{d g_{n}}{d t}(t)=g_{n}(t)+2 \sum g_{k}(t) \quad(0 \leqq k \leqq n-1),
$$

and a short computation reveals the important identity

$$
2 J D J^{-1}=(T+I)(T-I)^{-1}
$$

where

and

$$
(T f)(z)=z^{-1}(f(z)-f(0))
$$

$$
(D g)(t)=\lim _{h \rightarrow 0} h^{-1}(g(t+h)-g(t)) .
$$

This last limit is taken with respect to the norm topology on $L^{2}(0, \infty ; \mathscr{K})$. Since the open unit disc comprises the point spectrum of $T$, the right side of (2) is well defined on the range of $T-I$, a set whose closure is all of $H^{2}(\mathscr{K})$ because it contains every vector-valued polynomial

$$
p(z)=\varphi_{n} z^{n}+\varphi_{n-1} z^{n-1}+\cdots+\varphi_{1} z+\varphi_{0}
$$

with coefficients in $\mathscr{K}$.

If $A$ is an operator from $\mathscr{K}$ into $\mathscr{K}$, we designate its natural extension $\hat{A}$ on $H^{2}(\mathscr{K})$ by writing

$$
(\hat{A} f)(z)=\sum\left(A \varphi_{n}\right) z^{n}, \quad n=0,1,2, \cdots,
$$

whenever $f(z)=\sum \varphi_{n} z^{n}$. Clearly $\hat{A}$ commutates with $T$, and the substitution of (2) into (1) yields

$$
J L J^{-1}=\sum 2^{-k} \hat{A}_{k}(T+I)^{k}(T-I)^{-k} \quad(0 \leqq k \leqq n) .
$$


We infer from (3) that $L$ has a bounded inverse if and only if the operator

$$
S=(T-I)^{-n} \sum 2^{-k} \hat{A}_{k}(T+I)^{k}(T-I)^{n-k} \quad(0 \leqq k \leqq n)
$$

shares this property too.

A more effective method for determining the continuous invertibility of $S$ may be obtained by examining the adjoint operator $S^{*}$. Since

$$
\left(T^{*} f\right)(z)=z f(z) \text { and }\left(\hat{A}^{*} f\right)(z)=\sum\left(A^{*} \varphi_{n}\right) z^{n},
$$

a standard calculation involving the adjoint of a densely defined operator $[2$, p. 69] shows that

$$
\left(S^{*} f\right)(z)=R(z) f(z)
$$

where

$$
R(z)=\sum 2^{-k} A_{k}^{*}(z+1)^{k}(z-1)^{-k} \quad(0 \leqq k \leqq n) .
$$

According to a well-known result (Lemma 4.2 in [5]), $S^{*}$ has a bounded inverse if and only if there exists an analytic Toeplitz operator $Q(z)=$ $\sum Q_{n} z^{n}, n=0,1, \cdots$, defined on the interior of the unit disc such that

$$
R(z) Q(z)=Q(z) R(z)=I \text { and } \sup _{|z|<1}\|Q(z)\|<\infty .
$$

With this information at hand, it is possible to enunciate a simple invertibility criterion.

THEOREM. Let $\mathscr{K}$ be a separable Hilbert space, let $A_{0}, A_{1}, \cdots, A_{n}$ denote bounded linear operators from $\mathscr{K}$ into $\mathscr{K}$, and let $\mathscr{D}$ represent the set of all functions in $L^{2}(0, \infty ; \mathscr{K})$ whose first $n$ derivatives lie in $L^{2}(0, \infty ; \mathscr{K})$. Suppose further that $\mathscr{D}$ is endowed with the inner product inherited from $L^{2}(0, \infty ; \mathscr{K})$. Then the differential operator

$$
L=A_{n} \frac{d^{n}}{d t^{n}}+A_{n-1} \frac{d^{n-1}}{d t^{n-1}}+\cdots+A_{1} \frac{d}{d t}+A_{0}
$$

acting on $\mathscr{Z}$ has a bounded inverse if and only if the operator

$$
P(\sigma)=\sum A_{k}^{*} \sigma^{k} \quad(0 \leqq k \leqq n)
$$

acting on the Hilbert space $\mathscr{K}$ has a uniformly bounded inverse everywhere in the open half-plane $\operatorname{Re} \sigma<0$.

Proof. According to the arguments advanced before the derivation of (3), $L$ has a bounded inverse if and only if the operator $S$ enjoys the same property. It follows from elementary Hilbert space theory that $L$ has a bounded inverse if and only if the adjoint operator $S^{*}$ defined by (5) has a 
bounded inverse on $H^{2}(\mathscr{K})$. Moreover, since the linear fractional transformation $z=(2 \sigma+1)(2 \sigma-1)^{-1}$ maps the half-plane $\operatorname{Re} \sigma<0$ onto the disc $|z|<1$, we see from Rabindranathan's lemma that $S^{*}$ has a bounded inverse if and only if the operator-valued polynomial

$$
R\left(\frac{2 \sigma+1}{2 \sigma-1}\right)=\sum A_{k}^{*} \sigma^{k}=P(\sigma)
$$

has an analytic inverse which is uniformly bounded everywhere in the halfplane $\operatorname{Re} \sigma<0$. But the inverse of an operator-valued polynomial is clearly analytic, and this completes the proof.

A special case of our theorem deserves attention because of its utility in the study of matrix differential equations.

COROLLARY. When the dimension of $\mathscr{K}$ is finite, L has a bounded inverse if and only if the determinant of $P(\sigma)$ has no zeros in the closed left half-plane.

One final remark should be made at this point: It seems quite probable that our techniques can be extended to cope with differential equations having unbounded coefficients. Some results in this direction are now being prepared for later publication.

ACKNOWLEDGEMENT. The author is deeply indebted to the referee for catching an error in the original version of this note.

\section{REFERENCES}

1. A. Devinatz, Toeplitz operators on $H^{2}$ spaces, 'Trans. Amer. Math. Soc. 112 (1964), 304-317. MR 29 \#477.

2. E. R. Lorch, Spectral theory, University Texts in the Math. Sci., Oxford Univ. Press, New York, 1962. MR 25 \#427.

3. J. W. Moeller, On the differentiability and integrability of exponential sums, J. Math. Anal. Appl. 36 (1971), 301-307.

4. H. R. Pousson, Systems of Toeplitz operators on $H^{2}$. II, Trans. Amer. Math. Soc. 133 (1968), 527-536. MR 37 \#3377.

5. M. Rabindranathan, On the inversion of Toeplitz operators, J. Math. Mech. 19 (1969/70), 195-206. MR 40 \#4785.

6. M. Rosenblum, Self-adjoint Toeplitz operators, Summer Institute on Spectral Theory and Statistial Mechanics (Brookhaven National Laboratory), Upton, New York, 1965, pp. 135-157.

7. H. Widom, Inversion of Toeplitz matrices. II, Illinois J. Math. 4 (1960), 88-99. MR 24 \#A432.

Department of Mathematics, University of Illinois at Chicago Circle, Chicago, ILLINOIS 60680 